\title{
Phenotypic and genetic relationships between lamb and ewe traits for the Sabi sheep of Zimbabwe
}

\author{
O. Matika ${ }^{\dagger 1}$, J. B. van Wyk ${ }^{\# 1}$, G.J. Erasmus ${ }^{1}$ and R. L. Baker ${ }^{2}$ \\ ${ }^{1}$ Department of Animal Science, University of the Free State, P. O. Box 339, Bloemfontein 9300, Republic of South \\ Africa; ${ }^{2}$ International Livestock Research Institute P. O. Box 30709, Nairobi, Kenya.
}

\begin{abstract}
Genetic and phenotypic correlations were estimated between lamb and ewe traits in a flock of Sabi sheep reared at the Matopos Research Station. Direct additive estimates of heritability were: $0.28 \pm 0.04$; $0.17 \pm 0.00 ; 0.25 \pm 0.01 ; 0.39 \pm 0.01 ; 0.59 \pm 0.01 ; 0.50 \pm 0.01 ; 0.68 \pm 0.03 ; 0.12 \pm 0.003 ; 0.25 \pm 0.01 ; 0.11 \pm$ $0.00 ; 0.12 \pm 0.00$; for birth weight, weaning weight (120 days), 12 month weight, 18 month weight, ewe mating weight, post partum weight, ewe weight at weaning of lamb, total weight of lamb weaned, slaughter weight, hot and cold carcass weights respectively. Genetic correlations between birth weight and other weights to 18 months were high (0.75-0.85), whilst the relationship between weaning, 12 month and 18 month weight was close to unity. The genetic correlation between birth weight and ewe weights (mating, post-partum and dam weight at weaning of lamb) were moderate viz. $0.51 \pm 0.08,0.40 \pm 0.09,0.49 \pm 0.07$ respectively and were close to unity at 18 months of age viz. $0.96 \pm 0.02,0.92 \pm 0.03$, and $0.84 \pm 0.03$ respectively. Total weight of lamb weaned was moderately correlated to birth weight $\left(\mathrm{r}_{\mathrm{g}}=0.46 \pm 0.15\right)$ but tended to be highly correlated with 18 month weight $(0.92 \pm 0.10)$ and ewe weights $(0.75 \pm 0.09-0.91 \pm$ 0.07). The genetic correlation between birth weight and slaughter and carcass weight was moderate and was high at 18 months. The heritability estimates from a univariate logit transformed analysis for fertility, prolificacy and lamb survival were $0.08 \pm 0.04,0.22 \pm 0.03$, and $0.01 \pm 0.02$ respectively. The genetic correlation between fertility and lamb weight and ewe weights was low $(-0.08 \pm 0.004$, to $0.06 \pm 0.02)$ and some estimates had large standard errors. The genetic correlation between prolificacy and birth weight was close to zero. The genetic correlations between prolificacy and weaning weight, 12 month weight, 18 month weight, ewe mating, post-partum and ewe weight at weaning were $0.07 \pm 0.02 ; 0.12 \pm 0.00 ; 0.07 \pm 0.02 ; 0.22$ $\pm 0.00 ; 0.13 \pm 0.00 ; 0.24 \pm 0.00$ respectively, and that between fertility and prolificacy was negative $(-0.17 \pm$ 0.07). Genetic correlations between lamb survival and birth weight, weaning weight, 12 month weight, 18 month weight and total weight of lamb weaned were $0.18 \pm 0.00,0.26 \pm 0.00,0.15 \pm 0.00,0.15 \pm 0.00,0.13$ \pm 0.00 respectively. Selection for birth weight and total weight of lamb weaned have possible uses in a selection index for the Sabi flock.
\end{abstract}

Key words: Fat-tailed hair sheep, production, reproductive traits, lamb survival, correlations.

${ }^{\#}$ Corresponding author; e-mail: vanwykjb@ sci.uovs.ac.za

${ }^{\dagger}$ Present address: Matopos Research Station, P. Bag K5137, Bulawayo, Zimbabwe.

\section{Introduction}

Sabi sheep have hairy coats and fat tails and are suited to hot and semi-arid conditions (Mason, 1980; Fitzhugh \& Bradford, 1983). Sabi sheep have low birth and body weights under extensive production systems (Matika et al., 2001a). Selection for birth weight may be beneficial for Sabi sheep if genetic and phenotypic correlations are not antagonistic to other traits (Matika et al., 2001b). Breeding programmes designed to improve production efficiency require knowledge of genetic parameters for characters of economic importance such as growth rate, total weight of lamb weaned, prolificacy and reproduction. The aim of the current study was to estimate heritabilities and genetic and phenotypic correlations for growth, lamb survival and ewe traits for Sabi sheep reared in a semi-arid environment in order to formulate a breeding plan for this breed.

\section{Materials and Methods}

Data from Sabi sheep at the Matopos Research Station, Zimbabwe from 1984 to 1994 were used. The foundation flock was described by Ward (1959). From 1986, all ewes (400) were mated to 15 Sabi rams per mating cycle, and each sire was mated to 20-30 ewes. Two to three sires per year were used for repeat mating. Hand mating was done over a period of 35 days, and ewes lambed from the end of September until the 
beginning of December. Male single lambs with a birth weight of over $3 \mathrm{~kg}$ and male twins with a birth weight of over $2.5 \mathrm{~kg}$ were retained, and the rest of the male lambs were castrated. Weaning was done at 140 days of age up to 1990 and at 100 days of age thereafter. Maiden ewes were exposed to the rams at 18 months of age only if they had attained a minimum live weight of $30 \mathrm{~kg}$ and had no apparent deformities. All ewes that gave birth to twins and reared them to weaning were retained. Following selection of these animals, ewes that gave birth to single lambs and reared them to weaning and ewes that gave birth to twins and reared one to weaning were considered for selection. If more replacements were required, dry ewes and ewes which gave birth but did not wean a lamb were retained. Once the ewe flock had stabilised at about 450-500 ewes, i.e. from 1990 onwards, all dry ewes and those that did not rear lambs were culled. Animals with incomplete records of parentage were eliminated. Castrates, culled ewe lambs and culled rams were slaughtered between 18 and 30 months of age. A detailed description of management procedures is given by Matika et al. (2001a).

The following traits were analysed: birth weight within 24 hours of birth (BW); pre-weaning lamb survival (SURV); weaning weight (WW) at 120 days; 12 month weight (12W); 18 month weight (18W), total weight of lamb weaned (TWT); slaughter weight (SLW); hot carcass weight (HCW); cold carcass weight (CCW); ewe weight at mating (MW), post partum (PPW) and at weaning of lamb (EWW). Reproductive traits considered were fertility (FERT) (ewe lamb or not; 1 or 0 ) and prolificacy (PROL) (number of lambs born to ewes lambing; 1 or 2). A detailed description of the data used for reproductive parameters is given by Matika et al. (2001b). A description of the data used in the analysis is presented in Table 1.

Table 1 Summary of data used in this study

\begin{tabular}{lccc}
\hline Trait & Number & Mean & CV $(\%)$ \\
\hline & & & \\
Birth weight $(\mathrm{kg})$ & 4123 & 2.8 & 21.5 \\
Weaning weight $(\mathrm{kg})$ & 3537 & 17.8 & 18.0 \\
12 month weight $(\mathrm{kg})$ & 2219 & 23.9 & 15.2 \\
18 month weight $(\mathrm{kg})$ & 2035 & 34.4 & 20.4 \\
Slaughter weight $(\mathrm{kg})$ & 1635 & 35.8 & 24.4 \\
Hot carcass weight $(\mathrm{kg})$ & 1708 & 15.4 & 24.8 \\
Cold carcass weight $(\mathrm{kg})$ & 1708 & 15.0 & 28.0 \\
Total litter weight weaned ${ }^{*}(\mathrm{~kg})$ & 3318 & 19.6 & 11.8 \\
Ewe weight at mating $(\mathrm{kg})$ & 3654 & 37.9 & 12.9 \\
Ewe post partum weight $(\mathrm{kg})$ & 3724 & 35.4 & 10.0 \\
Ewe weight at weaning $(\mathrm{kg})$ & 2769 & 35.9 & 36.5 \\
Fertility & 4164 & 87.9 & 30.4 \\
Prolificacy & 3726 & 1.2 & 39.6 \\
Lamb Survival & 4353 & 85.6 & \\
\hline
\end{tabular}

* Sum of naturally reared individual lamb weights within a year per ewe lambing

Fixed effects included in the model were: year of birth (1984-1994), sex (males, castrates, females), type of birth (singles or twins), type of rearing, age of dam (2 to 7 years and older), birth date (measurement on day of birth nested within birth year for birth weight) and lamb age as a covariate for the rest of the traits. Year of birth and sex were combined into a single class to account for interaction after weaning because animals of different sexes were raised on separate farms.

Estimates of (co)variance components and breeding values were obtained using the ASREML programme (Gilmour et al., 1999) fitting bivariate animal models for all traits except for fertility, prolificacy and lamb survival. Reproductive traits and lamb survival were analysed using logit and probit link functions to link binomially distributed data to the normal distribution (Gilmour et al., 1999). Models fitted for the bivariate analyses were those determined from univariate analysis (Matika et al., 2001b). A model including direct and permanent environment due to the animal was fitted for fertility and prolificacy, while for lamb survival only direct additive effects were fitted. The results from logit and probit analyses were similar, and therefore only the heritability estimates from the logit analysis are presented. Genetic correlations between reproductive traits and lamb survival were done using Spearman's correlation of breeding values with the other traits. This should be considered an approximation. Although a genetic correlation is by definition a correlation between breeding values, the values used are a prediction. 


\section{Results and Discussion}

Heritability, phenotypic, environmental and genetic correlation estimates from bivariate analyses of growth, carcass, reproduction and lamb survival are presented in Table 2. Published genetic parameter estimates for different growth and reproduction traits are summarised in Tables 3 and 4.

Heritability estimates were in agreement with those of univariate analyses (Matika et al., 2001b), although they were slightly higher in some cases. This may be due to the fact that animals with both records were a selected sample and reproductive traits are measured in the female only. The subset of ewes that did not lamb is not a random sample of ewes, but a sample that has a lower than average ovulation rate (Waldron $\&$ Thomas, 1992). Direct heritability estimates were: $0.28,0.17,0.25,0.39,0.59,0.50,0.68,0.12,0.25,0.11$ and 0.12 for birth weight, weaning weight (120 days), 12 month weight, 18 month weight, ewe mating weight, post partum weight, ewe weight at lambing, total weight of lamb weaned, slaughter weight, hot and cold carcass weights respectively.

Genetic correlations between birth weight and other weights up to 18 months were high (0.75-0.85) whilst the relationship between weaning, 12 month and 18 month weight was close to unity. Fogarty (1995) reported lower genetic (0.07-0.32) and phenotypic correlations between birth weight and later weights. The largest relationships were found between chronologically adjacent weights, which is similar to other reports (Fogarty, 1995; Yazdi et al., 1997; El Fadili et al., 2000).

The genetic correlations between birth weights and ewe weights were moderate (mating, post-partum and dam weight at weaning of lamb were $0.51,0.40,0.49$ respectively) and those between 18 month weights and ewe weights were close to unity $(0.96,0.92$, and 0.84 respectively). The absence of genetic antagonisms between the various lamb and ewe weights indicate that none of the traits should be affected adversely by selection. Selecting for either birth weight or weaning weight without changing ewe weight would be difficult. This is in agreement with other reports (Nasholm \& Danell, 1996; Yazdi et al., 1997; Mousa et al., 1999).

Total weight of lamb weaned was moderately correlated to birth weight $\left(\mathrm{r}_{\mathrm{g}}=0.46\right)$, but tended to be highly correlated with 18 month weight (0.92) and ewe weight (0.75-0.91). Reported genetic and phenotypic correlations between live weights and total weight of lamb weaned are few and varied. Bromley et al. (2001) reported genetic correlations between birth weight and total weight of lamb weaned ranging from -0.22 to 0.28 and residual correlations of -0.02 to 0.00 (Table 3). Although their definition of total weight of lamb weaned was slightly different from that used in the current study, Snyman et al. (1998a,b) reported high (0.69-0.89) genetic and moderate (0.13-0.32) phenotypic correlations between total weight of lamb weaned and various lamb weights (Tables 3 and 4). In his review, Fogarty (1995) cited one reference for a genetic correlation between total weight of lamb weaned and hogget weight $(0.58)$ and a phenotypic correlation of 0.15 . In the same review, the correlation of total weight of lamb weaned with fertility was reported as 0.82 for genetic and $0.69-0.77$ for phenotypic correlations. Improvement of either 18-month weight or ewe weights would increase the total weight of lamb weaned. The genetic correlations between total weight of lamb weaned and fertility, prolificacy and lamb survival were $0.01,-0.05$ and 0.13 (Table 2), respectively and had large standard errors. These estimates were lower than those reviewed by Fogarty (1995) and others (Table 4). The genetic correlation between birth weight and slaughter and carcass weight was moderate and was high at 18 months. This is due to the fact that most of the males were slaughtered between 18 and 24 months of age.

The heritability estimates for fertility (0.08), prolificacy $(0.22)$ and lamb survival $(0.01)$ (Table 2) were from univariate, logit transformed analyses compared to estimates of $0.02,0.26$ and 0.04 , respectively reported for the same traits in the study using threshold models (Matika et al., 2001b). The estimate of heritability for fertility was higher than that from the threshold model, but still within the reported values (Matos et al., 1997, Olivier et al., 1998 (Grootfontein Merino stud)) but lower than 0.20 reported by Olivier et al. (1998)(Carnarvon Merino flock) and Snyman et al. (1998a). The estimate for prolificacy was almost the same as that reported for the threshold model using the same data set (Matika et al., 2001b). However, the estimate of heritability for survival (using logit transformation) was lower than that reported for the threshold model (Matos et al., 2000; Matika et al., 2001b) but was in close agreement to that reported elsewhere (Olivier et al., 1998; Snyman et al., 1998a; Lopez-Villalobos \& Garrick, 1999). 
Table 2 Heritability (on diagonal with SE in parenthesis), phenotypic (above diagonal and environmental correlations in parenthesis) and genetic correlation ${ }^{\dagger}$ (below diagonal with standard errors in parenthesis) estimates for growth, carcass and ewe traits

\begin{tabular}{|c|c|c|c|c|c|c|c|c|c|c|c|c|c|c|}
\hline Trait $^{\#}$ & BW & WW & W12 & W18 & MW & PPW & EWW & TWT & SLW & HCW & CCW & FERT & PROL & SURV \\
\hline $\mathrm{BW}$ & $\begin{array}{l}0.28 \\
(0.04)\end{array}$ & $\begin{array}{l}0.34 \\
(0.25)\end{array}$ & $\begin{array}{l}0.34 \\
(0.03)\end{array}$ & $\begin{array}{l}0.40 \\
(0.05)\end{array}$ & $\begin{array}{l}0.25 \\
(0.01)\end{array}$ & $\begin{array}{l}0.13 \quad(- \\
0.11)\end{array}$ & $\begin{array}{l}0.28 \\
(0.07)\end{array}$ & $\begin{array}{l}0.14 \\
(0.08)\end{array}$ & $\begin{array}{l}0.26 \\
(0.14)\end{array}$ & $\begin{array}{l}0.21 \\
(0.14)\end{array}$ & $\begin{array}{l}0.21 \\
(0.14)\end{array}$ & & & \\
\hline WW & $\begin{array}{l}0.81 \\
(0.07)\end{array}$ & $\begin{array}{l}\mathbf{0 . 1 7} \\
(\mathbf{0 . 0 0})\end{array}$ & $\begin{array}{l}0.80 \\
(0.78)\end{array}$ & $\begin{array}{l}0.66 \\
(0.61)\end{array}$ & $\begin{array}{l}0.55 \\
(0.32)\end{array}$ & $\begin{array}{l}0.48 \\
(0.19)\end{array}$ & $\begin{array}{l}0.50 \\
(0.13)\end{array}$ & $\mathrm{NC}$ & $\begin{array}{l}0.44 \\
(0.46)\end{array}$ & $\begin{array}{l}0.40 \\
(0.42)\end{array}$ & $\begin{array}{l}0.38 \\
(0.41)\end{array}$ & & & \\
\hline W12 & $\begin{array}{l}0.75 \\
(0.07)\end{array}$ & $\begin{array}{l}0.97 \\
(0.02)\end{array}$ & $\begin{array}{l}0.25 \\
(0.01)\end{array}$ & $\begin{array}{l}0.80 \\
(0.77)\end{array}$ & $\begin{array}{l}0.78 \\
(0.66)\end{array}$ & $\begin{array}{l}0.67 \\
(0.44)\end{array}$ & $\begin{array}{l}0.59 \\
(0.19)\end{array}$ & $\mathrm{NC}$ & $\begin{array}{l}0.59 \\
(0.44)\end{array}$ & $\begin{array}{l}0.54 \\
(0.45)\end{array}$ & $\begin{array}{l}0.53 \\
(0.45)\end{array}$ & & & \\
\hline W18 & $\begin{array}{l}0.85 \\
(0.05)\end{array}$ & $\begin{array}{l}0.94 \\
(0.03)\end{array}$ & $\begin{array}{l}0.94 \\
(0.02)\end{array}$ & $\begin{array}{l}0.39 \\
(0.01)\end{array}$ & $\begin{array}{l}0.90 \\
(0.74)\end{array}$ & $\begin{array}{l}0.81 \\
(0.45)\end{array}$ & $\begin{array}{l}0.71 \\
(0.36)\end{array}$ & $\begin{array}{l}0.61 \\
(0.03)\end{array}$ & $\begin{array}{l}0.77 \\
(0.70)\end{array}$ & $\begin{array}{l}0.71 \\
(0.62)\end{array}$ & $\begin{array}{l}0.70 \\
(0.61)\end{array}$ & & & \\
\hline MW & $\begin{array}{l}0.51 \\
(0.07)\end{array}$ & $\begin{array}{l}0.86 \\
(0.05)\end{array}$ & $\begin{array}{l}0.88 \\
(0.03)\end{array}$ & $\begin{array}{l}0.96 \\
(0.02)\end{array}$ & $\begin{array}{l}0.59 \\
(0.01)\end{array}$ & $\begin{array}{l}0.76 \\
(0.44)\end{array}$ & $\begin{array}{l}0.77 \\
(0.20)\end{array}$ & $\begin{array}{l}0.29 \\
(0.12)\end{array}$ & & & & & & \\
\hline PPW & $\begin{array}{l}0.40 \\
(0.08)\end{array}$ & $\begin{array}{l}0.86 \\
(0.05)\end{array}$ & $\begin{array}{l}0.88 \\
(0.03)\end{array}$ & $\begin{array}{l}0.92 \\
(0.031)\end{array}$ & $\begin{array}{l}0.96 \\
(0.008)\end{array}$ & $\begin{array}{l}0.50 \\
(0.01)\end{array}$ & $\begin{array}{l}0.74 \\
(0.30)\end{array}$ & $\begin{array}{l}0.31 \\
(0.13)\end{array}$ & & & & & & \\
\hline EWW & $\begin{array}{l}0.49 \\
(0.07)\end{array}$ & $\begin{array}{l}0.86 \\
(0.05)\end{array}$ & $\begin{array}{l}0.78 \\
(0.04)\end{array}$ & $\begin{array}{l}0.84 \\
(0.03)\end{array}$ & $\begin{array}{l}0.99 \\
(0.01)\end{array}$ & $\begin{array}{l}0.96 \\
(0.01)\end{array}$ & $\begin{array}{l}\mathbf{0 . 6 8} \\
(\mathbf{0 . 0 3})\end{array}$ & $\begin{array}{l}0.21 \\
(0.00)\end{array}$ & & & & & & \\
\hline TWT & $\begin{array}{l}0.46 \\
(0.15)\end{array}$ & $\mathrm{NC}^{*}$ & $\mathrm{NC}^{*}$ & $\begin{array}{l}0.92 \\
(0.09)\end{array}$ & $\begin{array}{l}0.83 \\
(0.07)\end{array}$ & $\begin{array}{l}0.91 \\
(0.06)\end{array}$ & $\begin{array}{l}0.75 \\
(0.08)\end{array}$ & $\begin{array}{l}0.12 \\
(0.00)\end{array}$ & & & & & & \\
\hline SLW & $\begin{array}{l}0.78 \\
(0.15)\end{array}$ & $\begin{array}{l}0.65 \\
(0.180)\end{array}$ & $\begin{array}{l}0.94 \\
(0.08)\end{array}$ & $\begin{array}{l}0.96 \\
(0.05)\end{array}$ & & & & & $\begin{array}{l}0.25 \\
(0.01)\end{array}$ & & & & & \\
\hline $\mathrm{HCW}$ & $\begin{array}{l}0.76 \\
(0.21)\end{array}$ & $\begin{array}{l}0.48 \\
(0.27)\end{array}$ & $\begin{array}{l}0.90 \\
(0.11)\end{array}$ & $\begin{array}{l}0.93 \\
(0.07)\end{array}$ & & & & & & $\begin{array}{l}0.11 \\
(0.00)\end{array}$ & & & & \\
\hline $\mathrm{CCW}$ & $\begin{array}{l}0.75 \\
(0.21)\end{array}$ & $\begin{array}{l}0.44 \\
(0.27)\end{array}$ & $\begin{array}{l}0.84 \\
(0.13)\end{array}$ & $\begin{array}{l}0.92 \\
(0.07)\end{array}$ & & & & & & & $\begin{array}{l}0.12 \\
(0.00)\end{array}$ & & & \\
\hline FERT & $\begin{array}{l}0.06 \\
(0.02)\end{array}$ & $\begin{array}{l}0.03 \\
(0.06)\end{array}$ & $\begin{array}{l}0.02 \\
(0.44)\end{array}$ & $\begin{array}{l}0.04 \\
(0.18)\end{array}$ & $\begin{array}{l}-0.08 \\
(0.00)\end{array}$ & $\begin{array}{l}0.01 \\
(0.62)\end{array}$ & $\begin{array}{l}-0.04 \\
(0.18)\end{array}$ & $\begin{array}{l}0.01 \\
(0.74)\end{array}$ & & & & $\begin{array}{l}\text { 0.08 } \\
(0.04)\end{array}$ & & \\
\hline PROL & $\begin{array}{l}-0.02 \\
(0.60)\end{array}$ & $\begin{array}{l}0.07 \\
(0.02)\end{array}$ & $\begin{array}{l}0.12 \\
(0.00)\end{array}$ & $\begin{array}{l}0.07 \\
(0.02)\end{array}$ & $\begin{array}{l}0.22 \\
(0.00)\end{array}$ & $\begin{array}{l}0.13 \\
(0.00)\end{array}$ & $\begin{array}{l}0.24 \\
(0.00)\end{array}$ & $\begin{array}{l}-0.05 \\
(0.07)\end{array}$ & & & & & $\begin{array}{l}0.22 \\
(0.03)\end{array}$ & \\
\hline SURV & $\begin{array}{l}0.18 \\
(0.00) \\
\end{array}$ & $\begin{array}{l}0.26 \\
(0.00) \\
\end{array}$ & $\begin{array}{l}0.15 \\
(0.00) \\
\end{array}$ & $\begin{array}{l}0.15 \\
(0.00) \\
\end{array}$ & $\begin{array}{l}0.05 \\
(0.06) \\
\end{array}$ & $\begin{array}{l}0.06 \\
(0.05) \\
\end{array}$ & $\begin{array}{l}0.03 \\
(0.25) \\
\end{array}$ & $\begin{array}{l}0.13 \\
(0.00) \\
\end{array}$ & & & & $\begin{array}{l}0.06 \\
(0.03) \\
\end{array}$ & $\begin{array}{l}0.14 \\
(0.00) \\
\end{array}$ & $\begin{array}{l}\mathbf{0 . 0 1} \\
(\mathbf{0 . 0 2}) \\
\end{array}$ \\
\hline
\end{tabular}

The South African Journal of Animal Science is available online at http://www.sasas.co.za/Sajas.html 
Table 3 A summary of published heritability $\left(h^{2}\right)$, phenotypic $\left(r_{p}\right)$, environmental $\left(r_{e}\right)$ and genetic correlation $\left(\mathrm{r}_{\mathrm{g}}\right)$ estimates for birth weight $(\mathrm{bw})$, weaning weight $(\mathrm{ww})$ and other parameters ${ }^{\#}$

\begin{tabular}{|c|c|c|c|c|c|c|}
\hline Breed & Trait2 & $\mathrm{h}^{2}($ Trait 1$)$ & $\mathrm{h}^{2}$ (Trait 2) & $r_{p}$ or $r_{e}^{*}$ & $\mathrm{r}_{\mathrm{g}}$ & Reference \\
\hline \multicolumn{7}{|l|}{ Birth weight } \\
\hline$\overline{\text { UAS Strain }}$ & w3 & 0.20 & 0.30 & 0.23 & -0.29 & Kumar \& Raheira, 1993 \\
\hline Suffolk & ww & 0.13 & 0.34 & 0.38 & 0.40 & Yamaki, 1994 \\
\hline Suffolk & w14 & 0.13 & 0.66 & 0.31 & 0.37 & Yamaki, 1994 \\
\hline Swedish finewool & ww & 0.06 & 0.15 & - & 0.44 & Nasholm \& Danell, 1996 \\
\hline Swedish finewool & slw & 0.06 & 0.15 & - & 0.44 & Nasholm \& Danell, 1996 \\
\hline Swedish finewool & mature & 0.07 & 0.63 & - & 0.36 & Nasholm \& Danell, 1996 \\
\hline Baluchi & WW & $0.14-0.20$ & $0.13-0.19$ & $0.39-0.41$ & $0.40-0.81$ & Yazdi et al., 1997 \\
\hline Segurena & ww & 0.43 & 0.31 & $0.51^{*}$ & 0.59 & Analla et al., 1997 \\
\hline Segurena & prol & 0.43 & 0.07 & $0.00^{*}$ & 0.18 & Analla et al., 1997 \\
\hline Segurena & w3 & 0.43 & 0.26 & $0.33^{*}$ & 0.56 & Analla et al., 1997 \\
\hline Composite & ww & 0.09 & 0.09 & 0.43 & 0.45 & Mousa et al., 1999 \\
\hline Composite & w19 & 0.09 & 0.35 & 0.27 & 0.35 & Mousa et al., 1999 \\
\hline Composite & w31 & 0.09 & 0.44 & 0.39 & -0.01 & Mousa et al., 1999 \\
\hline Moroccan Timahdit & w3 & 0.18 & 0.50 & 0.40 & 0.49 & El Fadili et al., 2000 \\
\hline Columbia & prol & 0.24 & 0.07 & $0.04^{*}$ & -0.01 & Bromley et al., 2000 \\
\hline Polypay & prol & 0.16 & 0.13 & $0.04^{*}$ & 0.03 & Bromley et al., 2000 \\
\hline Rambouillet & prol & 0.21 & 0.09 & $0.00^{*}$ & 0.26 & Bromley et al., 2000 \\
\hline Targhee & prol & 0.19 & 0.14 & $0.02^{*}$ & 0.11 & Bromley et al., 2000 \\
\hline Columbia & twt & 0.25 & 0.03 & $-0.02^{*}$ & -0.22 & Bromley et al., 2001 \\
\hline Polypay & twt & 0.16 & 0.09 & $0.00^{*}$ & 0.28 & Bromley et al., 2001 \\
\hline Rambouillet & twt & 0.20 & 0.14 & $-0.03^{*}$ & 0.23 & Bromley et al., 2001 \\
\hline Targhee & twt & 0.25 & 0.11 & $-0.02^{*}$ & 0.11 & Bromley et al., 2001 \\
\hline Dorper & ww & 0.11 & 0.20 & - & 0.27 & Neser et al., 2001 \\
\hline \multicolumn{7}{|l|}{ Weaning weight } \\
\hline Suffolk & w14 & 0.34 & 0.66 & 0.56 & 0.43 & Yamaki, 1994 \\
\hline Composite & w3 & 0.07 & 0.08 & 0.86 & 1.00 & Al-Shorepy \& Notter, 1996 \\
\hline Composite & w4 & 0.07 & 0.19 & 0.62 & 0.86 & Al-Shorepy \& Notter, 1996 \\
\hline Swedish finewool & slw & 0.14 & 0.18 & - & 0.95 & Nasholm \& Danell, 1996 \\
\hline Segurena & prol & 0.31 & 0.07 & $0.01^{*}$ & 0.48 & Analla et al., 1997 \\
\hline Segurena & w3 & 0.31 & 0.26 & $0.69^{*}$ & 0.76 & Analla et al., 1997 \\
\hline Baluchi & w12 & 0.19 & 0.32 & $0.59-0.60$ & $0.93-0.94$ & Yazdi et al., 1997 \\
\hline Afrino & w9 & 0.41 & 0.63 & 0.80 & 0.98 & Snyman et al., 1998a \\
\hline Afrino & w18 & 0.41 & 0.60 & 0.64 & 0.92 & Snyman et al., 1998a \\
\hline Afrino & twt & 0.41 & 0.17 & 0.13 & 0.75 & Snyman et al., 1998a \\
\hline Afrino & nlb & 0.41 & 0.23 & 0.04 & -0.01 & Snyman et al., 1998a \\
\hline Composite & w19 & 0.09 & 0.35 & 0.34 & 0.43 & Mousa et al., 1999 \\
\hline Composite & w31 & 0.09 & 0.44 & 0.30 & 0.32 & Mousa et al., 1999 \\
\hline Hungarian Merino & w12 & 0.21 & 0.13 & - & 0.54 & Nagy et al., 1999 \\
\hline Hungarian Merino & w24 & 0.21 & 0.11 & - & 0.41 & Nagy et al., 1999 \\
\hline Targhee & prol & 0.16 & 0.11 & 0.05 & 0.48 & Rao \& Notter, 2000 \\
\hline Suffolk & prol & 0.13 & 0.09 & 0.08 & 0.43 & Rao \& Notter, 2000 \\
\hline Polypay & prol & 0.10 & 0.10 & 0.02 & 0.09 & Rao \& Notter, 2000 \\
\hline
\end{tabular}

The genetic correlation between fertility and lamb and ewe weight was low (-0.08-0.06), and some estimates had large standard errors. This implies that the genes controlling fertility and live weight differ. The genetic correlation between prolificacy and birth weight was close to zero. Bromley et al. (2000) concluded that birth weight and prolificacy were only slightly genetically correlated, with mostly different genes involved in the expression of these two traits. The genetic correlations between prolificacy and weaning weight, 12 month weight, 18 month weight, ewe mating, post-partum and ewe weight at weaning were $0.07,0.12,0.07,0.22$, 0.13 and 0.24 respectively. Fogarty (1995) reported a weighted average genetic correlation between ewe 
weight and prolificacy at various ages of 0.41 (ranging between -0.46 and 0.78 ). The relationships between prolificacy and ewe weight vary and are reviewed by Michels et al. (2000). It appears that genetic improvement of ewe weight at mating or weaning will have a low to moderate response in improving prolificacy.

There is evidence of a small degree of genetic variation for lamb survival to weaning (Cundiff et al., 1982, Piper et al., 1982; Lopez-Villalobos \& Garrick, 1999; Matika et al., 2001b). Genetic correlations between lamb survival and birth weight, weaning weight, 12 month weight, 18 month weight and total weight of lamb weaned were $0.18,0.26,0.15,0.15,0.13$ respectively. However, the genetic correlations of preweaning lamb survival with ewe weights were low (0.03-0.06). Fogarty (1995) reviewed lamb survival as a ewe trait and its genetic correlation ranged from $0.16,0.11,0.51$ to -0.30 for birth weight, weaning, hogget weight and total weight of lamb weaned respectively. There were also low phenotypic correlations varying from -0.18 to 0.04. Selection for live weight in the Sabi flock will not improve survival to a great extent but will have some beneficial effects. Better control of environmental effects will result in higher lamb survival.

Table 4 Summary of published heritability $\left(h^{2}\right)$, phenotypic $\left(r_{p}\right)$, environmental $\left(r_{e}\right)$ and genetic correlation $\left(r_{g}\right)$ estimates for post weaning growth, reproduction traits and other parameters ${ }^{\#}$

\begin{tabular}{|c|c|c|c|c|c|c|c|}
\hline Breed & Trait1 & Trait2 & $\begin{array}{l}\mathrm{h}^{2} \text { Trait } \\
1\end{array}$ & $\begin{array}{l}\mathrm{h}^{2} \text { Trait } \\
2\end{array}$ & $r_{p}$ or $r_{e}^{*}$ & $r_{g}$ & Reference \\
\hline \multicolumn{8}{|l|}{ Prolificacy } \\
\hline Rambouillet & prol & w6 & 0.19 & 0.35 & $0.03^{*}$ & 0.22 & Waldron \& Thomas, 1992 \\
\hline Composite & prol & w3 & 0.05 & 0.14 & 0.11 & 0.33 & Al-Shorepy \& Notter, 1996 \\
\hline Composite & prol & fert & 0.05 & 0.09 & - & 0.56 & Al-Shorepy \& Notter, 1996 \\
\hline Segurena & prol & w3 & 0.07 & 0.26 & $0.01^{*}$ & 0.36 & Analla et al., 1997 \\
\hline Rambouillet & prol & w18 & 0.06 & 0.48 & $0.00^{*}$ & 0.35 & Lee et al., 2000 \\
\hline Columbia & prol & twt & 0.07 & 0.02 & $0.41^{*}$ & 0.65 & Bromley et al., 2001 \\
\hline Polypay & prol & twt & 0.12 & 0.07 & $0.33^{*}$ & 0.42 & Bromley et al., 2001 \\
\hline Rambouillet & prol & twt & 0.09 & 0.10 & $0.33^{*}$ & 0.62 & Bromley et al., 2001 \\
\hline Targhee & prol & twt & 0.10 & 0.10 & $0.33^{*}$ & 0.55 & Bromley et al., 2001 \\
\hline \multicolumn{8}{|c|}{ Total weight of lamb weaned } \\
\hline Afrino & twt & w18 & 0.17 & 0.60 & 0.26 & 0.89 & Snyman et al., 1998a \\
\hline Tygerhoek Merino & twt & w14-16 & 0.13 & 0.55 & 0.15 & 0.80 & Snyman et al., 1998 b \\
\hline Grootfontein Merino & twt & w14-16 & 0.13 & 0.38 & 0.20 & 0.67 & Snyman et al., $1998 \mathrm{~b}$ \\
\hline $\begin{array}{l}\text { Klerefontein Merino } \\
\text { Fertility }\end{array}$ & twt & w14-16 & 0.22 & 0.43 & 0.32 & 0.72 & Snyman et al., 1998b \\
\hline $\begin{array}{l}\text { Composite } \\
\text { Others }\end{array}$ & fert & w3 & 0.09 & 0.14 & -0.04 & -0.31 & Al-Shorepy \& Notter, 1996 \\
\hline Swedish Finewool & mature & slw & 0.39 & 0.24 & - & 0.44 & Nasholm \& Danell, 1996 \\
\hline Various & w12 & w18 & 0.26 & 0.36 & 0.71 & 0.62 & Stobart et al., 1986 \\
\hline Various & w12 & w30 & 0.26 & 0.25 & 0.55 & 0.24 & Stobart et al., 1986 \\
\hline Various & w18 & w30 & 0.36 & 0.25 & 0.75 & 0.73 & Stobart et al., 1986 \\
\hline Afrino & w18 & nlb & 0.60 & 0.23 & 0.16 & 0.31 & Snyman et al., 1998a \\
\hline Composite & w19 & w31 & 0.35 & 0.44 & 0.65 & 0.97 & Mousa et al., 1999 \\
\hline Hungarian Merino & w12 & w24 & 0.13 & 0.11 & & 0.57 & Nagy et al., 1999 \\
\hline
\end{tabular}

\# other parameters: $w 3=90$ day weight; $w 6=180$ day weight; $w 12=12$ month weight; $w 14=14$ month weight; $w 16=$ 16 month weight; w18 = 18 month weight; w19 = 19 month weight; w24 = 24 month weight; w30 = 30 month weight; w31 = 31 month weight, twt = total weight of lamb weaned; slw = slaughter weight; fert = fertility; prol = prolificacy; nlb $=$ number of lambs born.

\section{Conclusions}

It was concluded that antagonistic relationships did not exist between the traits examined. The positive genetic correlation between birth weight and survival suggests that this flock is below the optimum; this could be due to a gradual deterioration in the environment. Contrary to the case with improved livestock, where a policy of guarding against high birth weights is generally recommended, genetic improvement of birth weight, also because of its high genetic correlation with later weights, should not be avoided in the Sabi sheep until an optimum is reached. Total weight of lamb weaned should also be included in the selection strategy since it is a 
composite trait that incorporates elements of lamb growth and survival to weaning and ewe reproductive performance.

\section{References}

Al-Shorepy, S. A. \& Notter, D. R., 1996. Genetic variation and covariation for ewe reproduction, lamb growth and lamb scrotal circumference in a fall-lambing sheep flock. J. Anim. Sci. 74, 1490-1498.

Analla, M., Munoz-Serrano, A. \& Serradilla, J. M., 1997. Analysis of the genetic relationship between litter size and weight traits in Segurena sheep. Can. J. Anim. Sci. 77, 17-21.

Bromley, C. M., Snowder, G. D. \& Van Vleck, L. D., 2000. Genetic parameters among weight, prolificacy and wool traits of Columbia, Polypay, Rambouillet and Targhee sheep. J. Anim. Sci. 78, 846-858.

Bromley, C. M., Van Vleck, L. D. \& Snowder, G. D., 2001. Genetic correlations for litter weight weaned with growth, prolificacy and wool traits in Columbia, Polypay, Rambouillet and Targhee sheep. J. Anim. Sci.79, 339-346.

Butcher, R. L., Dunbar, R. S. Jr. \& Welch, J. A., 1964. Heritabilities of and correlations between lamb birth weight and 140-day weight. J. Anim. Sci. 23, 12-15.

Cundiff, L. V., Gregory, K. E. \& Koch, R. M., 1982. Selection for increased survival from birth to weaning. Proc. $2^{\text {nd }}$ World Congr. Gen. Appl. Livest. Prod. V, 310-337.

Davis, G. H., Morris, C. A. \& Dodds, K. G., 1998. Genetic studies of prolificacy in New Zealand sheep. Anim. Sci. 67, 289-297.

Dickerson, G. E., 1970. Efficiency of animal production - molding the biological components. J. Anim. Sci. 30, 849-859.

El Fadili, M., Michaux, C., Detilleux, J. \& Leroy, P. L., 2000. Genetic parameters for growth traits of the Moroccan Timahdit breed of sheep. Small Rumin. Res. 37, 203-208.

Fitzhugh, H. A. \& Brandford, G. E., 1983. Hair sheep of western Africa and the Americas: A genetic resource for the tropics. Westview Press, boulder, Co.

Fogarty, N. M., 1995. Genetic parameters for live weight, fat and muscle measurements, wool production and reproduction in sheep: a review. Anim. Breed. Abstr. 63, 101-143.

Gilmour, A. R., Cullis, B. R., Welham, S. J. \& Thompson, R., 1999. ASREML- Reference manual. NSW Agriculture Biometric Bulletin No. 3. NSW Agriculture, Orange Agricultural Institute, Forest Road, Orange 2800, NSW, Australia.

Kumar, N. \& Raheja, K. L., 1993. Genetic and phenotypic parameters of growth and reproduction traits in UAS strain of sheep estimated by multitrait animal model. Indian J. Anim. Sci. 63, 978-983.

Lee, J. W., Waldron, D. F. \& Van Vleck, L. D., 2000. Parameter estimates for number of lambs born at different ages and for 18-month body weight of Rambouillet sheep. J. Anim. Sci.78, 2086-2090.

Lopez-Villalobos, N. \& Garrick, D. J., 1999. Genetic parameter estimates for lamb survival in Romney sheep. Proc. N. Z. Soc. Anim. Prod. 59, 121-124.

Mason, I. L. 1980. Prolific tropical sheep. FAO Animal Production and health paper 17, 1.

Matika, O., Van Wyk, J.B., Erasmus, G.J. \& Baker, R.L., 2001a. A desciption of growth, carcass and reproductive traits of Sabi sheep in Zimbabwe. Small Rumin. Res. (submitted)

Matika, O., Van Wyk, J.B., Erasmus, G.J. \& Baker, R.L., 2001b. Genetic parameter estimates in Sabi sheep. Livest. Prod. Sci.. (submitted)

Matos, C.A.P, Thomas, D.L., Gianola, D., Tempelman, R.J. \& Young, L.D., 1997. Genetic analysis of discrete reproductive traits in sheep using linear and non-linear models: I. Estimation of genetic parameters. J. Anim. Sci. 75, 76-87.

Matos, C. A. P., Thomas, D. L. Young, L. D. \& Gianola, D., 2000. Genetic analysis of lamb survival in Rambouillet and Finnsheep flocks by linear and threshold models. Anim. Sci. 71, 227-234.

Michels, H., Decuypere, E. \& Onagbesan, O., 2000. Litter size, ovulation rate and prenatal survival in relation to ewe body weight:genetics review. Small Rumin. Res. 38, 199-209.

Mousa, E., van Vleck, L. D. \& Leymaster, K. A., 1999. Genetic parameters for growth traits for a composite terminal sire breed of sheep. J. Anim. Sci. 77, 1659-1665.

Morris, C. A., Hickey, S. M. \& Clarke, J. N., 2000. Genetic and environmental factors affecting lamb at birth and through to weaning. N. Z. J. Agric. Res. 43, 515-524.

Nagy, I., Solkner, J., Komlosi, I. \& Safar, L., 1999. Genetic parameters of production and fertility traits in Hungarian Merino sheep. J. Anim. Breed. Genet. 116, 399-413. 
Nasholm, A. \& Danell, O., 1996. Genetic relationships of lamb weight, maternal ability and mature ewe weight in Swedish finewool sheep. J. Anim. Sci. 74, 329-339.

Neser, F W. C., Erasmus, G. J. \& Van Wyk, J. B., 2001. Genetic parameter estimates for pre-weaning weight traits in Dorper sheep. Small Rumin. Res. 40, 197-202.

Olivier, W.J., Snyman, M. A., Van Wyk, J.B., Erasmus, G.J., 1998. Genetic parameter estimates for fitness traits in South African Merino. Livest. Prod. Sci. 56, 71-77.

Piper, L. R., Hanrahan, J. P., Evans, R., Bindon, B. M., 1982. Genetic variation in individual and maternal components of lamb survival in Merinos. Proc. Aust. Soc. Anim. Prod. 14, 29-30.

Rao, S. \& Notter, D. R., 2000. Genetic analysis of litter size in Targhee, Suffolk, and Polypay sheep. J. Anim. Sci 78, 2113-2120.

Snyman, M. A., Erasmus, G. J., Van Wyk, J. B. \& Olivier, J. J., 1998a. Genetic and phenotypic correlations among production and reproduction traits in Afrino sheep. S. Afr. J. Sci. 28, 74-81.

Snyman, M. A., Cloete, S. W. P. \& Olivier, J. J., 1998b. Genetic and phenotypic correlations of total weight of lamb weaned with body weight, clean fleece weight and mean fibre diameter in three South African Merino flocks. Livest. Prod. Sci. 55, 157-162.

Stobart, H. R., Bassett, J. W., Cartwright, T. C. \& Blackwell, R. L., 1986. An analysis of body weights and maturing patterns in western range ewes. J. Anim. Sci. 63, 729-740.

Waldron, D. F. \& Thomas, D. L., 1992. Increased litter size in Rambouillet sheep: 1. Estimation of genetic parameters. J. Anim. Sci. 70, 3333-3344.

Yamaki, K., 1994. Growth of lambs and genetic parameters in four breeds. Proc. $5^{\text {th }}$ World Congr. Gen. Appl. Livest. Prod.18, 135-138.

Yazdi, M. H., Engstrom, G, Nasholm, A., Johansson, K., Jorjani, H. \& Liljedahl, L. -L, 1997. Genetic parameter for lamb weight at different ages and wool production in Baluchi sheep. Anim. Sci. 65, 247255. 